\title{
Implementing Health Behavior Change in Primary Care: Lessons From Prescription for Health
}

Deborab J. Coben, $\mathrm{PbD}$
Alfred F. Tallia, $M D, M P H$

Benjamin F. Crabtree, $P b D$

Denise M. Young, MD

Department of Family Medicine, University of Medicine and Dentistry, Robert Wood Johnson Medical School, Somerset, NJ
Conflicts of interest: none reported

\section{CORRESPONDING AUTHOR}

Deborah J. Cohen, PhD

Department of Family Medicine

University of Medicine and Dentistry

Robert Wood Johnson Medical School

1 Worlds Fair Dr

Somerset, NJ 08803

debbiec@nac.net

\begin{abstract}
PURPOSE Our objective was to identify themes that emerged from the evaluation of 17 interventions funded by the Robert Wood Johnson Foundation's Prescription for Health that aimed to enhance adherence to healthy behaviors in the primary care setting.
\end{abstract}

METHODS We performed a content analysis of diary data from this 16-month initiative. Other data sources used to complement this analysis include funded grant applications and field notes from interviews with investigative teams and a limited number of site visits. Participants were 17 practice-based research networks (PBRNs) that had projects funded during Round 1 of Prescription for Health.

RESULTS Five themes emerged regarding implementation of health behavior change: (1) health behavior change resources are enthusiastically received by practices and patients, and when given a choice, patients prefer methods of assistance that involve personal contact; (2) practice extenders require extensive training, as well as careful case management and support, in order to function fully and avoid burnout; (3) integrating behavior change tools into the primary care setting requires time, effort, and often specialized expertise; (4) even simple interventions require practice change, and use of a practice change model to guide implementation efforts is crucial; and (5) research philosophy and project management approaches vary across PBRNs and have implications for the potential sustainability of an intervention.

CONCLUSIONS A more versatile, multifaceted solution involving new tools, technologies, and multidisciplinary care teams is needed in order to integrate health behavior change into everyday primary care routines. Even the best interventions require a model to articulate how to integrate an innovation into practices.

Ann Fam Med 2005;3(Suppl 2):S12-S19. DOI: 10.1370/afm.334.

\section{INTRODUCTION}

7 here is widespread acknowledgment that fundamental changes are required in primary care practices to meet the diverse care needs of patients, families, and communities in the United States. ${ }^{1,2}$ This need for change is particularly evident in targeting lifestyle issues that are the leading causes of morbidity and mortality nationally. ${ }^{3}$ Although innovative models and practical tools and techniques have been developed to address lifestyle issues in primary care practices, these have not been well disseminated. There is a growing recognition that the ecology of primary care practice is complicated ${ }^{4}$ and that clinician behavior is relatively resistant to common approaches to change. ${ }^{5-7}$ To facilitate change in primary care, greater attention to adoption, implementation, and maintenance, ${ }^{2}$ practice capacity, ${ }^{8,9}$ and health care system linkages ${ }^{10}$ is needed.

Prescription for Health: Promoting Healthy Behaviors in Primary Care Research Networks is a national program of the Robert Wood Johnson Foundation (RWJF) in collaboration with the Agency for Healthcare 
Research and Quality (AHRQ). This program provides 2 rounds of grant funding to primary care practicebased research networks (PBRNs) to develop and pilot-test creative practical strategies for integrating approaches to health behavior change into routine primary care practice. The program targets 4 health risk behaviors that are the nation's leading causes of preventable disease, disability, and premature death: lack of physical activity, poor diet, tobacco use, and risky use of alcohol. ${ }^{11}$ This report focuses on Round 1 of the Prescription for Health initiative, which ended October 2004. Round 2 begins in July 2005.

The RWJF established the Prescription for Health Analysis Team (A-Team) to conduct an independent process evaluation of the program. The goals of this evaluation were to understand project and practice characteristics that support the successful implementation of and adherence to healthy behavior interventions in the primary care setting, and to identify new insights and patterns that transcend individual projects. In this article, we describe 5 cross-cutting themes that emerged from our analysis of evaluation data collected during Round 1 of the initiative. These themes underscore the need for approaches to health behavior change in the primary care setting, and they identify the types of resources and capacities necessary to develop and integrate health behavior interventions in this setting.

\section{METHODS}

\section{Data Collection}

The A-Team performed a multimethod assessment that included collecting survey data at the PBRN and practice levels, as well conducting interviews and a limited number of site visits with each investigative team (Figure 1). Although these data provided cross-sec-

\section{Figure 1. RWJF Prescription for Health evaluation plan.}

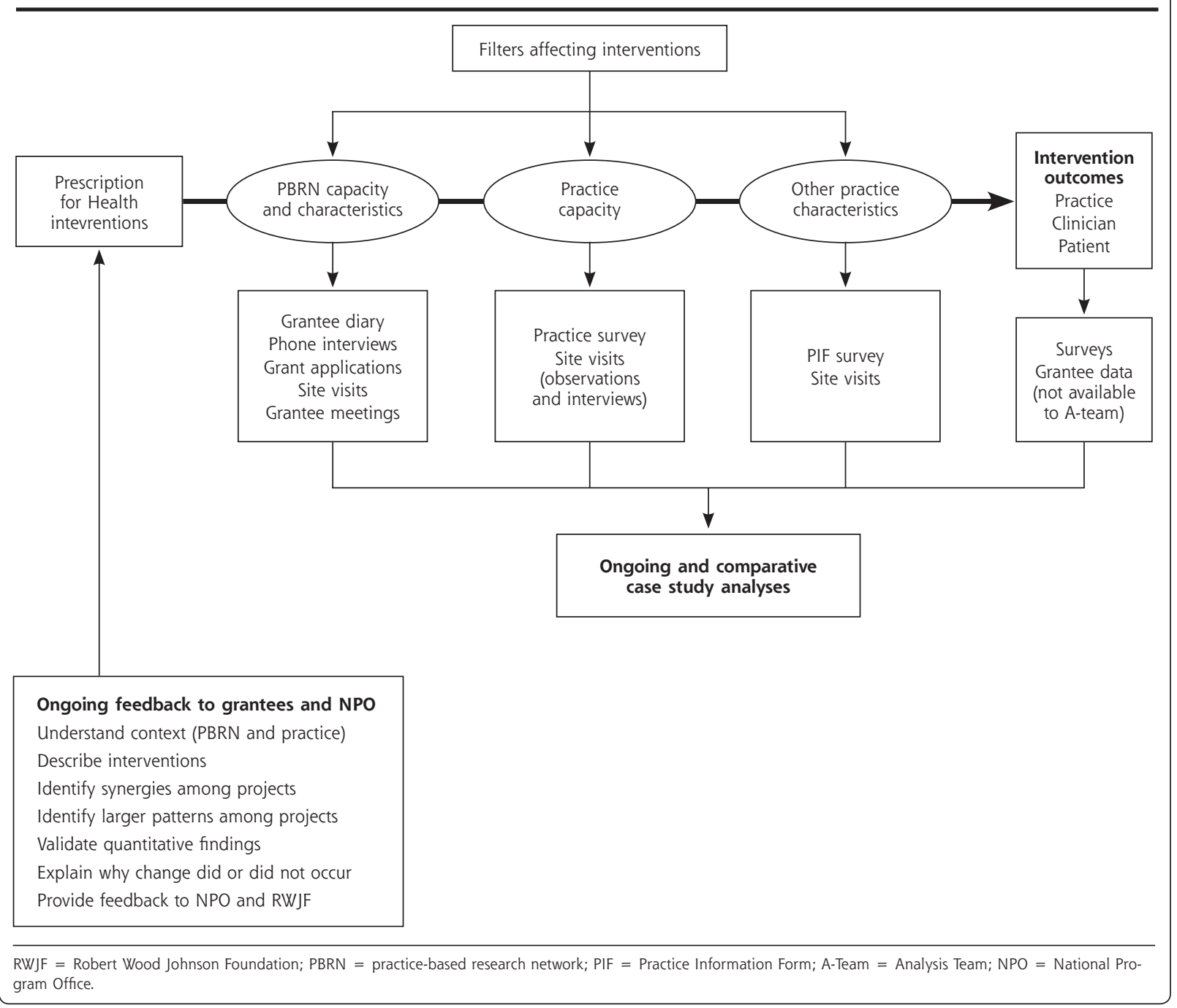


tional PBRN-, practice-, and project-level information that allowed ongoing insight into the implementation process, interactive online project diary "rooms" were created on the AHRQ-sponsored PBRN secure extranet for each of the 17 investigative teams. Project diaries functioned as both a data collection tool, providing additional insight into the implementation process, and as a mechanism for facilitating ongoing 2-way communication between investigative teams and the A-team.

The A-Team conducted a content analysis of all funded grant applications to determine the scope and focus of each project, and to identify potential diary keepers from each investigative team. In June 2003, the team sent a personalized letter to each principal investigator outlining the details of the evaluation and identifying research team members who were potential diary keepers. During a 30-minute telephone call with each investigative team, 3 to 5 people were selected to be diary keepers, at least 1 or 2 of whom would be in close contact with practices during the implementation process. Diary keepers were asked to make an entry twice a month. Questions to help spark the diary-writing process were provided via a help button in each diary room, but in general, diary keepers were asked to write about their project development, recruitment, and implementation experiences. The A-Team regularly posted responses to diary entries to elicit more information or elaboration from a diary keeper.

Two members of the A-Team received e-mail notification each time a new diary entry was posted. One team member read diary entries at the time of receipt, addressed immediate concerns, and copied the entry into a word-processing program to facilitate sharing with A-Team members.

\section{Analyses}

\section{Real-Time Process Analysis}

The A-Team conducted a real-time process analysis that involved the ongoing iterative process of reading and reflecting on the data as they were collected. ${ }^{12}$ During weekly meetings lasting approximately 3 hours, each diary entry was read aloud and discussed. Other data (eg, survey data, notes from interviews and site visits), as well as earlier diary postings, were brought in as needed to fully understand the implementation experiences reported. Decisions were made regarding how to respond to the week's diary entries, and 1 person posted questions to each diary room. Using data from the project diaries, as well as notes from key interviews and site visits, the A-Team created case descriptions for each project and updated these descriptions regularly as new data were received. Grant applications, diary entries, and field notes taken during the site visit were incorporated into a com- prehensive searchable Folio Views database (ver 4.11, Open Market Inc, Burlington, Mass).

\section{Comprehensive Analysis}

In April 2004, when many projects were well under way and diary contributions were substantial, the ATeam transitioned into a single case analysis mode in which all available data for a project (eg, diaries, grant applications, interview and site visit notes, and communications, as well as available survey data) were examined in greater detail. This analysis proceeded iteratively and involved an immersion-crystallization approach. ${ }^{12,13}$ First, the A-Team examined data to identify overarching, organizing themes. Subsequently, A-Team members were asked to take the lead on identifying, describing, and articulating project themes via the development of a preliminary report. In September 2004, preliminary summary reports were created and an in-depth cross-case comparison was conducted to identify cross-cutting themes. ${ }^{12,14}$ To accomplish this task, A-Team members read each preliminary report independently, looking for common patterns across projects and working to reach consensus in regard to the key implementation lessons. This process involved reexamining relevant data within and across projects when discrepancies arose, and corresponding with grantees to collect additional data, and to confirm or refute insights or themes, particularly when themes were present in 1 project but absent in others. At the culmination of Round 1, the A-Team prepared a final report for each investigative team that included an analysis and synthesis of the findings.

\section{RESULTS}

Fifty-seven grantees were asked to keep online diaries, and 569 diary entries were made. The median number of monthly diary entries made per project team was between 3 and 4 . The A-Team conducted site visits with 9 PBRNs and conducted 2 interviews with each investigative team.

Round 1 Prescription for Health projects represent a diverse array of intervention approaches (Table 1). In the sections that follow, we describe the 5 themes that emerged from our analysis of the evaluation data collected from this round of the initiative.

\section{Theme 1: The Need for Health Behavior Change Interventions}

Practices and patients enthusiastically respond to interventions that provide an additional health behavior change resource; practices eagerly refer patients to this resource, and patient recruitment is easy, with referrals often exceeding what research teams can offer. 


\begin{tabular}{|c|c|}
\hline Project & Intervention Description \\
\hline 1 & $\begin{array}{l}\text { Prescription pad to trigger clinician referral to established wellness program that } \\
\text { provided telephone support (physical activity and smoking cessation). }\end{array}$ \\
\hline 2 & $\begin{array}{l}\text { Interactive educational program based on } \mathrm{Ml} \text {; practice member screened and } \\
\text { identified eligible patients; patients selected intervention intensity (written } \\
\text { material, Web site, telephone counseling). }\end{array}$ \\
\hline 3 & $\begin{array}{l}\text { Standardized paper-based practice assessments; patients received pedometers, } \\
\text { nutrition and activity logs, referral to a walking club, and coaching. }\end{array}$ \\
\hline 4 & $\begin{array}{l}\text { PDA-guided clinical assessment of patients' eating and smoking behaviors using a } \\
5 \mathrm{~A}^{\prime} \text { s model; patient referral to a health coach for support. }\end{array}$ \\
\hline 5 & $\begin{array}{l}\text { Health system nurse-consultant facilitated quality improvement effort to enhance } \\
\text { delivery and documentation of } 5 A^{\prime} \text { s. }\end{array}$ \\
\hline 6 & $\begin{array}{l}\text { Tailored practice improvement to increase pediatrician counseling on diet and } \\
\text { physical activity. Practices selected options of proven prevention strategies. }\end{array}$ \\
\hline 7 & $\begin{array}{l}\text { PDA risk survey for teens; triggers clinicians to initiate } \mathrm{MI} \text { and action plan; follow- } \\
\text { up e-mail support. }\end{array}$ \\
\hline 8 & $\begin{array}{l}\text { Wireless Web tablet used to assess patients' concerns (diet and activity); triggers } \\
\text { discussion; patients offered group counseling. }\end{array}$ \\
\hline 9 & $\begin{array}{l}\text { Practice protocol to identify overweight and obesity; referral to a health coach for } \\
\text { MI-based program. }\end{array}$ \\
\hline 10 & $\begin{array}{l}\text { Comparative analysis of (1) using innovative Web-based patient activation tool, } \\
\text { (2) using Web tool plus linkages to a practice improvement approach, (3) using } \\
\text { Web tool and linkages to the practice improvement approach plus practice link- } \\
\text { ages to community resources. }\end{array}$ \\
\hline 11 & $\begin{array}{l}\text { Stage-based Web portal for patients to access evidence-based resources on health } \\
\text { behaviors (tools to facilitate change, community services information, assistance } \\
\text { in arranging help from practices). }\end{array}$ \\
\hline 12 & $\begin{array}{l}\text { Practice members engaged in a personal behavior change for improving diet and } \\
\text { physical activity; offer same intervention (goal-setting, pedometers, telephone } \\
\text { follow-up) to patients. }\end{array}$ \\
\hline 13 & $\begin{array}{l}\text { Searchable Web-based Community Health Promotion Resource; assessed impact } \\
\text { of this tool on clinician discussions of health behavior change, patient's readi- } \\
\text { ness to change, and actual change. }\end{array}$ \\
\hline 14 & $\begin{array}{l}\text { Goal setting with patients who were overweight and at risk for diabetes; practice } \\
\text { protocol developed to identify eligible patients; patients received pedometer } \\
\text { and were monitored by nurse surveillance. }\end{array}$ \\
\hline 15 & $\begin{array}{l}\text { PDA-based decision support tool to improve clinicians' ability to provide patient- } \\
\text { tailored counseling (tobacco, diet) at point of care; practice-developed clinician } \\
\text { trigger for PDA use. }\end{array}$ \\
\hline 16 & $\begin{array}{l}\text { Tested penetration of } 3 \text { different brief intervention models (clinician, specialist, } \\
\text { health educator) for smoking and risky drinking; practice-tailored assessment } \\
\text { tools; clinician training. }\end{array}$ \\
\hline 17 & $\begin{array}{l}\text { Clinicians integrated behavior change action plans into routine visits; research staff } \\
\text { members assisted with patient recruitment, follow-up calls, and record reviews. }\end{array}$ \\
\hline
\end{tabular}

clinic the staff wanted to enroll as study participants! (Project 2, diary entry, 1/8/04)

Entbusiasm is running very bigh. At one site, 7 of 12 signed up for a mini-triatblon-_all for the 1st time... They put a map on the wall, got some clip art, and the teams are marching across the countrypassing each other up. (Project 12, diary entry, $12 / 24 / 03$ )

\section{Patients' Enthusiasm}

The second group met one week later. Eleven of 12 families returned. They are all very entbusiastic. They brought their babit books which they bad kept pretty meticulously.

(Project 8 , diary entry, 1/29/04)

We are also experiencing profound disappointment expressed by patients who do not qualify for the study...many patients are desperately seeking belp. (Project 14, diary entry, 2/13/04)

\section{Patients' Preference for Personal Contact} We bave no one that has selected the selfbelp condition, as of yet... Telephone is the more popular choice at this point. (Project 2, diary entries, 2/17/04 and $2 / 19 / 04)$

\section{Theme 2: Training and Supporting Practice Extenders}

Several projects used practice members or practice extenders such as lay coaches or nonclinical peers to deliver health behavior counseling. One benefit of this approach was that moderate to intensive health

The data suggest that many patients want someone to talk with when undertaking a health behavior change, and when given options, patients choose intervention approaches that facilitate interaction with a counselor or coach rather than those that are self-guided. The following diary entries illustrate these observations.

\section{Practices' Enthusiasm}

They bave been very cooperative and are looking forward to the intervention which they think is really needed at their site considering that "we feel unprepared to assess and manage overweight children." (Project 8, diary entry, 10/24/03)

From the largest to the smallest clinic, the physicians and site coordinators are thrilled to be a part of the study and ask if they can begin recruiting immediately after our visit. In fact, at one behavior counseling could be offered while minimizing the impact on practice routines and resources. Our analysis highlights another benefit. In the context of practice extender-patient relationships, patients may share personal stories around behavior change, and for some doing so may be important to the change process. In one project, the intensity and depth of personal experiences patients shared was surprising to investigators and may have arisen from the nonthreatening and supportive relationship the practice extenders created. For example, a diary keeper noted the following:

My follow-up phone contact with the client on Friday was intense and lengtby (45 $\mathrm{min})$. She began sobbing within one minute of my open-ended question, "How bas your week been?" I also had a long telephone interview with a medical student who bas 
struggled with obesity and suffered bulimia since age 12. She spoke eloquently about the internal workings of overweight folks in ber experience. She spoke of a kind of numbness that feels both good and bad. That was new to me. She talked about shame and depression. (Project 9, diary entry, 9/19/03)

Although physician extenders relieve practices of work involved in facilitating and supporting health behavior change in patients, resources are needed to support physician extenders financially and provide extensive and continuous training. In addition, patients and extenders would benefit from access to a local care team (eg, clinicians, psychologists, social workers) for followup, consultation, and referral for treatment of issues that surface during counseling sessions, such as depression. One physician extender's diary entries suggests that she felt inadequately trained and supported and, after several months of working with patients, began to experience burnout, reporting that she felt exhausted having "less compassion for the patients" and trying "to continue to be cordial and empathetic, but the energy demanded of contacts drained me" (Project 9, diary entry, 7/6/04).

\section{Theme 3: Adapting Tools and Techniques to Primary Care Practice}

Tools and techniques used in Prescription for Health interventions include Web-based resources, handheld computers or personal digital assistants (PDAs), email follow-up, action plans and forms, lay coaches/ community health associates, statewide and local telephone counseling, group visits, pedometers and walking groups, and prescription pads. Many of these approaches were not specifically developed for practice settings and, therefore, did not automatically fit well. For example, research teams modified action plan forms to fit with clinical practice (Project 17, field notes, $1 / 18 / 04)$, and another project tailored their patient coaching strategy to suit practice staffing patterns and work demands (Project 12, field notes, 3/31/04).

Although all projects managed to develop and integrate their behavior change tools, all seriously underestimated the time and effort needed to integrate and adapt these approaches to the primary care setting. For some, mobilization of other resources provided economic and intellectual support critical to this effort. Tool development was a labor-intensive process involving both special technical expertise (eg, software developers) and an understanding of the practice environment. For example, a Project 11 diary entry reported a list of information technology activities ranging from having meetings with computer programmers to discuss the big picture, as well as specifics on Internet service provider (ISP) algorithms, to registering domain names, obtaining permission from the Outreach Office to use an external domain to point to a university server, setting up Web space on a secure server, testing file transfer protocol (FTP) software and a beta version of the Web site, and so forth. Other projects noted similar activities:

Computer support feels using the Palm bas its advantages as a collection device, but we've learned through experience that remote support of users is not easy. You bave to deal with installation of Palm software on remote PCs, installation and support of the program on the Palm (and there's little control of what else the practices put on the machine), network issues, and general end-user support. (Project 7, diary entry, 10/10/03)

Software issues were discussed and the final copy edits are being completed on the Palm version. The PC version is also getting closer to completion. Final testing will be performed prior to deployment. (Project 15, diary entry, 5/21/04-9 months into Round 1)

Developing tools that rely on computer-based technologies requires the early involvement of experts (eg, computer programmers, designers), not only to design, develop, and customize these tools, but also to work with the research team to develop realistic timelines that include time for pilot- and beta-testing. Integrating a new tool or approach into a primary care practice (computer-based or not) requires a good understanding of the practice's systems. The collection of practice-level data aimed at identifying systems of organization and routine care processes in practices would help researchers tailor their tools to better fit the practice setting.

\section{Theme 4: Using an Implementation Model to Guide Practice Change}

Most Prescription for Health interventions asked practices to make substantial changes to their organization, including modifications to their work routines and systems of care (eg, referring to a new program, using a new tool or protocol, engaging in action planning). We examined grant applications, site visit notes, and diary data to look for references or allusions to characteristics identified in the literature as important for integrating innovations into primary care practices for sustainable change, such as stakeholder motivation, leadership, role of local champions, practice capacity and resources for change, external environment, and perceived benefit. ${ }^{15,16}$ We found that few projects used a formal change model or proactively considered these characteristics.

Project diary entries highlight the potential consequences of this omission. For example, the need to consider stakeholder motivation and perceived benefit was apparent when an innovation (eg, PDAs, Web-based resources) was designed to replace an existing process that practices perceived as satisfactory. Project 13 diary entries (1/14/04 and 2/2/04) chronicle the many telephone calls and personal visits involved in recruiting practices. In more than 1 practice, the Web-based 
system the researchers offered seemed to be replacing a paper-based educational system, and clinicians did not appear eager to make this change. To encourage this transition, the research team attempted to tailor the new system to the practices by offering paper versions of educational materials posted on the Web:

Practices are selecting some materials that they would like to bave on band for their education files. Stacey is checking and requesting permission again from each source and being sure that the source is appropriately cited on each document. (Project 13, diary entry, 2/18/04)

Similar difficulties were observed for nontechnological interventions. As the following entry suggests, clinicians may be motivated to enhance preventive service delivery, but they may not see the value in changing aspects of their existing system:

Dr. stated be was quite confident be is addressing all behaviors and 5 A's thoroughly and was satisfied to remain status quo. He stated be did use catch-all phrases like lifestyle modification as documented evidence and assurance that be is addressing all bebaviors and believes that be would be "Covered in a court of law." (Project 5, diary entry, 1/1/04)

Additionally, contact with research teams was required for problem-solving, and in many cases investigators needed to work with practice leaders and administrators to mobilize the resources necessary to implement an intervention. The following entries describe these interactions:

We are trying to work out a process that will allow the clinics to incorporate the action plan process into their regular routine after we are gone. (Project 17, diary entry, 9/24/03)

Biller receives request for chart identification. Spends several bours in one day trying to meet request and cannot. Doctor notes that this is too costly. Requests to be dropped from study. I follow up with doctor. "If we can get the PHO (Pbysician Health Organization) to do this part for you would that make it ok?" "Much better." (Project 5, diary entry, 11/11/03)

All the clinicians that have been enrolled seem excited about the program, but I know from personal experience (since we at the University bave had access to the program) that it is very easy to forget to actually make the referral. We are currently discussing ways to keep the $\mathrm{P}_{4} \mathrm{H}[$ Prescription for Health] program on the minds of the clinicians. (Project 1, diary entry, 1/7/04)

Although research teams were flexible when tailoring interventions to practices, this process was commonly problem-focused and reactive. There is little evidence to suggest use of a formal organizational change model to guide these efforts proactively.

\section{Theme 5: PBRN Research Philosophy and Project Management Approach}

Our data suggested that several participating PBRNs, particularly those with a long history of successful PBRN research, had well-developed strategies for generating enthusiasm for participation in studies and managing the research projects conducted in their PBRN. There was evidence that this extensive research experience, as well as a history of conducting projects with finite endpoints (eg, card studies and chart audit surveys), may have socialized research teams and member practices into a "project mentality," leading them to regard interventions as finite research projects that have definable endpoints rather than improvements that may become a permanent part of the practice organizational design. Such experience and views are evident in the following entries:

This clinic will be upgrading their status in the PBRN from ruby to diamond as they participate in the $\mathrm{P}_{4} \mathrm{H}$ project. This is especially good news as it represents their willingness to assume more family practice based research projects in this clinic. (Project 2 , diary entry, $11 / 17 / 03$ )

The doctor was interested in the project and expressed a bit of concern on the side of the physicians since they see 36 patients a day each pbysician. He mentions that the "demand for patient care is so bigh... Only 36 patients will need follow up calls (2 more) until the study ends. ..."At the end of the conversation, be supported the idea bebind this project and agreed that be will supervise the progress of this project until the end of the study. (Project 12, diary entry, 5/24/04; A-Team emphasis added)

As usual, we bave some "producers" and some "non-producers" in the clinics. Thanks goodness for the real time recruitment Web site. Because of it I have been able to identify and call all of the clinics who are bebind schedule. (Project 2, diary entry, 4/26/04)

The idea that there are producers and nonproducers in terms of practice recruitment, and that practices just need to make it until the end of the study suggests that PBRN researchers and practice members view their implementation of interventions as a short-term obligation, not necessarily as a long-term practice improvement. Fostering this kind of project approach or project mentality may well be counterproductive if a study seeks to stimulate the practice to make sustained changes to its existing care processes. In fact, there are a number of diary entries, such as those above, that appear to set up an expectation among physicians that the goal of the research is to enroll a set number of patients and that this goal was all that was expected for participation. Bodenheimer et al ${ }^{17}$ further discuss the tensions between PBRN research and the realities of practice.

\section{DISCUSSION}

The RWJF's Prescription for Health initiative demonstrates the enormous opportunity that exists to foster health behavior change and underscores the critical role primary care practices can and should play in the 
widespread delivery of these services. We nevertheless uncovered the unanticipated difficulties encountered by some projects when attempting to implement their interventions, as well as the apparent lack of sustainability of these projects in the practice setting. Our analysis suggests 3 steps that may improve the feasibility and sustainability of PBRN research around health behavior interventions.

First, research team members need a strong understanding of the organizational features of practice that have been shown to mediate successful implementation of practice change interventions. In Round 1 of Prescription for Health, several well-crafted, potentially useful innovations had poor uptake, and others were too resource-intensive for practices to implement without external assistance. Understanding practice organization and using a practice change model to inform study design can proactively cultivate research projects that are better aligned with the needs, resources, and capacities of primary care practices. ${ }^{18-21}$

Second, PBRNs and research teams attempting to develop strategies for promoting healthy behaviors in the primary care setting need to develop collaborative multidisciplinary teams that bring together experts in such areas as information technology, patient care and counseling, community outreach, clinician education, and practice organization and change. The collaborative effort of such experts is needed to develop realistic and comprehensive interventions for health behavior change that not only help clinicians identify patients who need to change and are ready (eg, for interventions involving PDAs, Web-based systems), but also assist clinicians when discussing behavior change with patients (eg, for interventions involving action planning, PDA-driven protocols) and provide the counseling and support needed to make and sustain such changes (eg, for interventions involving individual and group coaching and counseling, telephone counseling). Multidisciplinary teams need to think about how to combine the functionality of several of the interventions tested under this initiative, and how to do this in a way that is both feasible and sustainable at the practice level.

Third, as more PBRNs gain experience in conducting research, PBRN leaders need to reflect on their philosophy and approach for managing the research process. The steps PBRNs may take to motivate practices, to ease the burden a research project places on a practice, and to develop organizational systems for managing multiple projects simultaneously may inadvertently detract from the basic purpose of PBRNs-to provide a research laboratory that embodies real-life practice. Additionally, although some projects may be endpoint-oriented, others may need to be handled differently and in ways that foster long-term sustainable quality improvements. PBRN leaders and researchers need to be aware of how their handling of a research project may have implications for how the practice treats an intervention, for example, whether it is viewed as a short-term or a long-term change.

Our study is not without its limitations. The data we collected as part of our evaluation were not consistent across all projects. Each project had different research goals, and study designs varied greatly. As a result, comparing similar experiences or identifying themes that arose across all projects was difficult. When possible, we took steps to fill these gaps. Additionally, although some projects were very generous in taking the time to make detailed and insightful diary entries, others were less inclined to do so. The depth of our understanding of a project's implementation experience depended in large part on the quantity and quality its diary entries, and there was great variation across projects and across diary keepers. We moderated the potential impact of this limitation by taking steps to encourage teams to make diary entries and by soliciting experiences among similar sets of projects to ascertain a wide range of experiences around an emergent theme.

To read or post commentaries in response to this article, see it online at http://www.annfammed.org/cgi/content/full/3/Suppl_2/S12.

Key words: Behavior; health behavior; health promotion/disease prevention; practice-based research; practice of medicine; health care delivery; primary care

Submitted January 18, 2005; submitted, revised, April 13, 2005; accepted April 15, 2005.

Funding support: This work was supported by Prescription for Health, a national program of The Robert Wood Johnson Foundation (grant No. 47075) with support from the Agency for Healthcare Research and Quality, and by a grant from the Health Resource Services Administration (grant 5 T32 HP10011-12-00).

\section{References}

1. Institute of Medicine. Crossing the Quality Chasm: A New Health System for the 21st Century. Washington, DC: National Academies Press; 2001.

2. Glasgow RE, Orleans CT, Wagner EH. Does the Chronic Care Model serve also as a template for improving prevention? Milbank $Q$. 2001;79:579-612, iv-v.

3. McGinnis J, Williams-Russo P, Knickman J. The case for more active policy attention to health promotion. Health Aff (Millwood). 2002;21:78-93.

4. Stange KC. The paradox of the parts and the whole in understanding and improving general practice. Int J Qual Health Care. 2002;14:267268.

5. Eisenberg JM. Physician utilization: the state of research about physicians' practice patterns. Med Care. 1985;23:461-483.

6. Greco PJ, Eisenberg JM. Changing physicians' practices. N Engl J Med. 1993;329:1271-1273. 
7. Berwick DM. Disseminating innovations in health care. JAMA. 2003;289:1969-1975.

8. Cohen D, McDaniel RR Jr, Crabtree BF, et al. A practice change model for quality improvement in primary care practice. J Healthc Manag. 2004;49:155-168.

9. Woolf SH. Taking critical appraisal to extremes: the need for balance in the evaluation of evidence. J Fam Pract. 2000;49:1081-1085.

10. Grol R. Improving the quality of medical care: building bridges among professional pride, payer profit, and patient satisfaction. JAMA. 2001;286:2578-2585.

11. Mokdad A, Marks J, Stroup D, Gerberding J. Actual causes of death in the United States. JAMA. 2004;291:1238-1245.

12. Miller WL, Crabtree BF. The dance of interpretation. In: Crabtree BF, Miller WL, eds. Doing Qualitative Research. 2nd ed. Thousand Oaks, Calif: Sage Publications; 1999:127-143.

13. Borkan J. Immersion/crystallization. In: Crabtree BF, Miller WL, eds. Doing Qualitative Research. 2nd ed. Thousand Oaks, Calif: Sage Publications; 1999:179-194.

14. Crabtree BF, Miller WL, eds. Doing Qualitative Research. 2nd ed. Thousand Oaks, Calif: Sage Publications; 1999.
15. Rogers EM. Diffusion of Innovations. 5th ed. New York, NY: Free Press; 2003.

16. Bradley E, Webster T, Baker D, et al. Translating Research Into Practice: Speeding the Adoption of Innovative Health Care Programs. New York, NY: Commonwealth Fund; 2004.

17. Bodenheimer T, Young DM, MacGregor K, Holtrop JS. Practice-based research in primary care: facilitator of or barrier to practice improvement? Ann Fam Med. 2005;3(Suppl):p-p.

18. Grol R, Wensing M. What drives change? Barriers to and incentives for achieving evidence-based practice. Med J Aust. 2004;180(6 Suppl):S57-S60.

19. Grol R, Grimshaw J. From best evidence to best practice: effective implementation of change in patients' care. Lancet. 2003;362:12251230.

20. Halm EA, Siu AL. Are quality improvement messages registering? Health Serv Res. 2005;40:311-316.

21. Tierney WM, Overhage JM, Murray MD, et al. Can computer-generated evidence-based care suggestions enhance evidence-based management of asthma and chronic obstructive pulmonary disease? A randomized, controlled trial. Health Serv Res. 2005;40:477-498. 